\title{
Fast simulation of Time-of-Flight detectors at the LHC
}

\author{
Olivier Rousselle ${ }^{1, *}$ and Tom Sykora ${ }^{2, * *}$ \\ ${ }^{1}$ Laboratoire Kastler Brossel, Sorbonne Université, ENS-PSL, Collège de France, CNRS, Paris, France \\ ${ }^{2}$ Institute of Particle and Nuclear Physics, Faculty of Mathematics and Physics, Charles University, \\ Prague, Czech Republic
}

\begin{abstract}
The modelling of Cherenkov based detectors is traditionally done using Geant4 toolkit. In this work, we present another method based on Python programming language and Numba high performance compiler to speed up the simulation. As an example we take one of the Forward Proton Detectors at the CERN LHC - ATLAS Forward Proton (AFP) Time-of-Flight, which is used to reduce the background from multiple proton-proton collisions in soft and hard diffractive events. We describe the technical details of the fast Cherenkov model of photon generation and transportation through the optical part of the ToF detector. The fast simulation is revealed to be about 200 times faster than the corresponding Geant 4 simulation, and provides similar results concerning length and time distributions of photons. The study is meant as the first step in a construction of a building kit allowing creation of a fast simulation of an arbitrary shaped optical part of detectors.
\end{abstract}

Keywords: Geant4 - Python/Numba - HEP - Cherenkov detector

\section{Introduction}

Geant4 is a Monte Carlo toolkit to simulate the passage and interactions of elementary particles with matter [1]. Geant 4 simulation has been used to optimize the optical shape and performance of Time-of-Flight detector $[2,3]$. In this modelling, a run corresponds to set of proton trajectories going through the detector, an event corresponds to one proton passing through the detector, a track corresponds to a photon emitted from a vertex of the trajectory, and a step corresponds to a photon trajectory from one side to another side of the detector. However, Geant 4 simulation of Cherenkov photons is very time-consuming: the simulation of a thousand events may take few hours when using the full physics list [2]. We expect the geometrical method presented here to be faster than Geant4 [4]. The fast Cherenkov model is based on efficient photon path length calculations, which use the laws of geometrical optics and some approximations. It uses the programming language Python [5] and the Numba module (a Python high performance compiler) to speed up the process [6].

\footnotetext{
*e-mail: olivier.rousselle@cern.ch

**e-mail: tomas.sykora@cern.ch
} 


\section{Example of study: ATLAS Forward Proton ToF detector}

\subsection{Characteristics of ToF detector}

The ATLAS Forward Proton (AFP) detector aims at measuring diffractive protons leaving under very small angles (order of hundreds of $\mu \mathrm{rad}$ ) the ATLAS interaction point (IP) [2]. The AFP stations are placed $205 \mathrm{~m}$ and $217 \mathrm{~m}$ in both directions from the ATLAS IP (fig. 1).

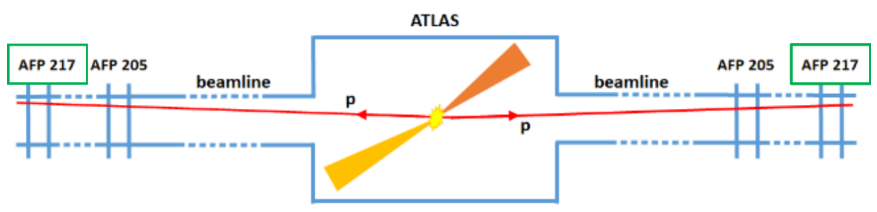

Figure 1. Position of AFP detectors in ATLAS experiment.

The far stations (noted by green boxes in fig.1) consist of two subdetectors: Silicon Tracker (SiT) and ToF detector. The optical part of the ToF detector is composed of 16 "L-shaped" silica bars ( $4 \times 4$ LQbars); those bars are rotated by $48^{\circ}$ with respect to the LHC beam [7]. The set of 4 bars in the horizontal row is called a train. Trains are numbered 1,2,3,4; for each train, bars are denoted with letters A,B,C,D (see fig.2). Note also that train 1 has a functional improvement in design, called "taper", whose purpose is to straighten the photon trajectories and thus detect them in the photomultiplier (PMT) in the shortest possible time [7]. In fig.2, we also present the coordinate system used (with orthonormal basis $x, y, z$ ).

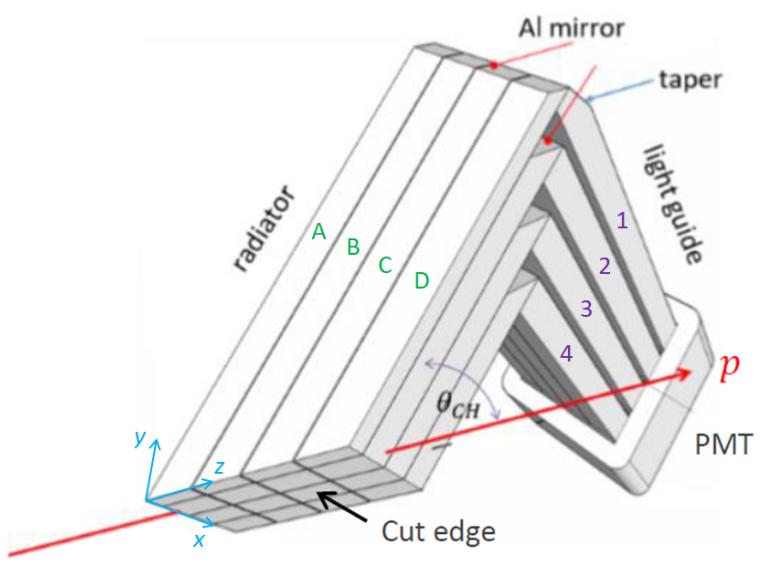

Figure 2. Optical part of the ToF detector [8].

\subsection{Generation of protons and Cherenkov photons}

In our study, we consider proton trajectories parallel to the cut edge (see fig.2). Protons crossing the silica material (Suprasil) composing the ToF detector go faster than light, and 
emit Cherenkov radiation. The average number of photons generated by a proton is given by the formula $[8,9]$ :

$$
\mu \approx 370 \cdot Z^{2} \cdot \text { Steplength } \cdot \int_{\mathrm{E}_{\min }}^{\mathrm{E}_{\max }}\left(1-\frac{1}{\beta^{2} n^{2}(E)}\right) d E
$$

where $Z=q / e=1$ for protons; StepLength is the length of a proton trajectory in the Cherenkov active material; $\beta=v_{\mathrm{p}} / c \approx 1$ is the relativistic factor of a proton (it propagates nearly at the speed of light in the considered application); $E$ is the energy of a photon corresponding to wavelength in the range accepted by the photomultiplier; $n$ is the refraction index of silica (depending on photon wavelength).

Along the proton trajectory, photons are emitted from vertices, denoted as $A\left(x_{A}, y_{A}, z_{A}\right)$. Within the fast simulation, a wavelength is attributed randomly (in the range $[180 \mathrm{~nm}$; 650nm]) to each photon following the Cherenkov distribution [8]. The Cherenkov angle $\theta_{\text {ch }}$ depends on the refraction index $n$ and thus on the wavelength of the photon $\lambda$. Its value for $\lambda \sim 400 \mathrm{~nm}$ is: $\theta_{\mathrm{ch}}=\arccos \left(\frac{1}{\beta n}\right) \approx 48^{\circ}$ for silica [10]. Each photon is generated randomly (uniformly) with an angle $\varphi \in[-\pi, \pi]$, indicating the direction of the light ray inside the Cherenkov cone (fig.3).

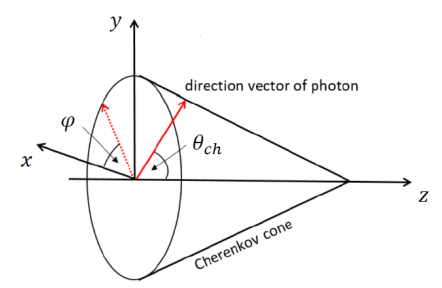

Figure 3. Illustration of Cherenkov cone [8].

Photons travel through the radiator, the light guide and finally to a photomultiplier (PMT). Transmission through the LQBars can be viewed in the approximation of geometrical optics, with internal reflections and losses [10].

\subsection{Edge effect}

Depending on the proton trajectory and on the values of $\theta_{\mathrm{ch}}$ and $\varphi$, a photon can reflect from the cut edge (represented in fig.2), which leads to the so-called the edge effect. In fig.4, we illustrate examples of trajectories when the photon doesn't touch the cut edge (orange line), touches the cut edge and remains in the same bar (blue line), or touches the cut edge and goes to another bar (green line). 


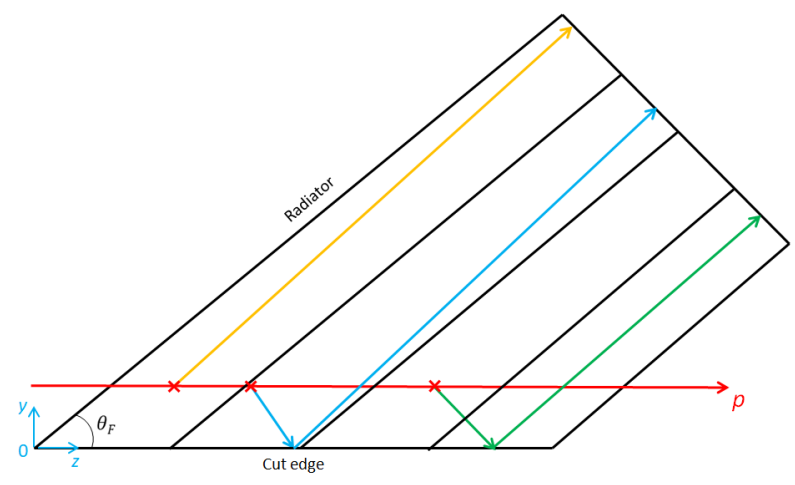

Figure 4. Illustration of different photon trajectories in a train (view in 2D).

\section{Fast simulation}

\subsection{Fast simulation flow}

We present here the different steps of the fast simulation:

Generation of proton trajectories

$\downarrow$
Generation of Cherenkov photons

$\downarrow$

Photon tracking through the ToF detector (radiator and light guide)

$\downarrow$

Photons reach the photomultiplier (PMT)

\subsection{Possible cases}

We distinguish 4 different geometrical cases for the trajectory of a photon:

- Case 0: photon doesn't reach the PMT;

- Case 1: photon moves via the path $A \rightarrow$ Aluminium (Al.) mirror $\rightarrow$ PMT;

- Case 2: photon moves via the path $A \rightarrow$ PMT (without touching Al. mirror);

- Case 3: photon moves via the path $A \rightarrow$ cut edge $\rightarrow$ PMT.

\subsection{Attenuation effects}

In whichever the case, the absolute majority of photons bounce on the sides of the radiator and the sides of the light guide. Depending on the incident angle, a photon can be reflected or not. The following attenuation effects are implemented in the fast simulation [8,9]:

- Reflectivity on the Al. mirror (90\%);

- Each time the light ray hits a side (bound) and satisfies the conditions of total reflection, we use $99 \%$ as the value of probability of reflection;

- Attenuation in the material (silica), which is function of the wavelength of the photon. The probability of absorption is given by: $p_{\text {abs }}=1-e^{\mu_{\text {abs }} L}$ with $\mu_{\text {abs }}=\frac{1}{L_{\text {abs }}}$ the attenuation coefficient of the material [4]. $L_{\mathrm{abs}}$ is the attenuation length which is a function of wavelength; its evolution is taken from [11]. 
If the conditions of reflection through its full path are satisfied and the photon is not absorbed, it will reach by the PMT.

\subsection{Track length and time}

We denote $L$ the length of photon track between the vertex $A$ and the PMT ( $L=L_{\mathrm{A} \rightarrow \mathrm{PMT}}$ ). The value of the length depends on the case considered. For example, for the case 1 [12]:

$$
L=L_{\mathrm{A} \rightarrow \text { Mirror }}+L_{\text {Mirror } \rightarrow \text { PMT }}=\frac{L_{\mathrm{rad}}-y_{\mathrm{A}}}{\cos \left(\alpha_{1}\right) \cdot \cos \left(\delta_{1}\right)}+\frac{L_{\mathrm{lg}}}{\cos \left(\alpha_{2}\right) \cdot \cos \left(\delta_{2}\right)}
$$

with $L_{\mathrm{rad}} / L_{\mathrm{lg}}$ the lengths of the radiator/light guide, and $\alpha_{1}, \delta_{1}, \alpha_{2}, \delta_{2}$ the reflection angles. The time-of-flight of the photon, from the vertex point to the PMT is given by:

$$
t_{\gamma}=\frac{L}{c / n}
$$

The time includes the time a proton spends when travelling from $t=0$ to the vertex $A$ (with length noted $L_{\mathrm{p}}$ ), and the time of the photon (from vertex $A$ to the PMT):

$$
t=t_{\mathrm{p}}+t_{\gamma}=\frac{L_{\mathrm{p}}}{c}+\frac{L}{c / n}
$$

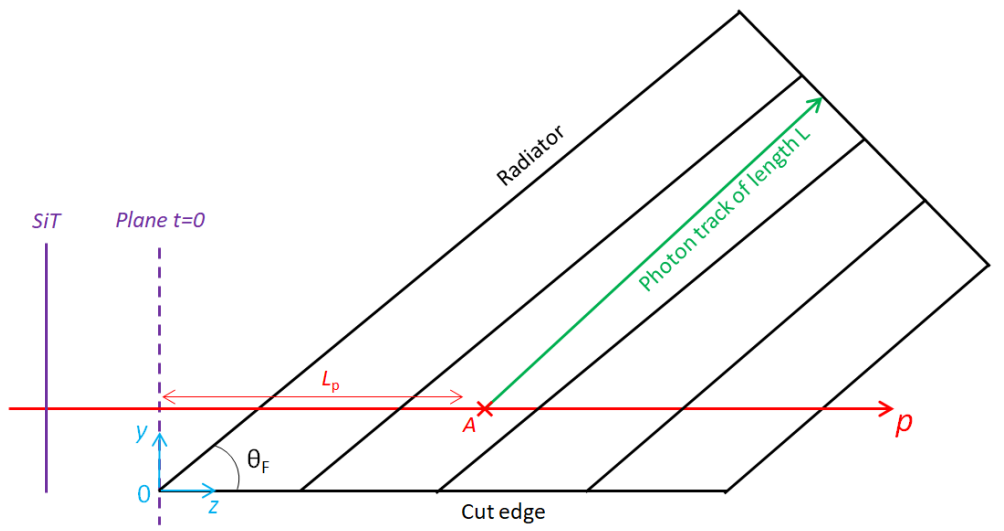

Figure 5. Illustration of time measurement from the vertical plane $(t=0)$ to the PMT.

\section{Fast simulation vs Geant 4}

\subsection{Comparison of length and time distributions for the case of a full train}

In order to compare the results of the fast simulation and Geant4, we performed the simulation by generating $10^{5}$ Cherenkov photons with random positions of vertices, for a fixed trajectory of proton. We represent in fig. 6 the distributions of lengths and times obtained for train 2 . About $43 \%$ of initial photons reach the PMT, and the percentages of geometrical cases are $42 \%$ case $1 ; 19 \%$ case $2 ; 39 \%$ case 3 .

The distributions have very similar shapes, and the relative error is less than $2 \%$ (in absolute value) for $95 \%$ of photon tracks (see fig.7). The fast simulation is then in a good agreement with Geant4. 

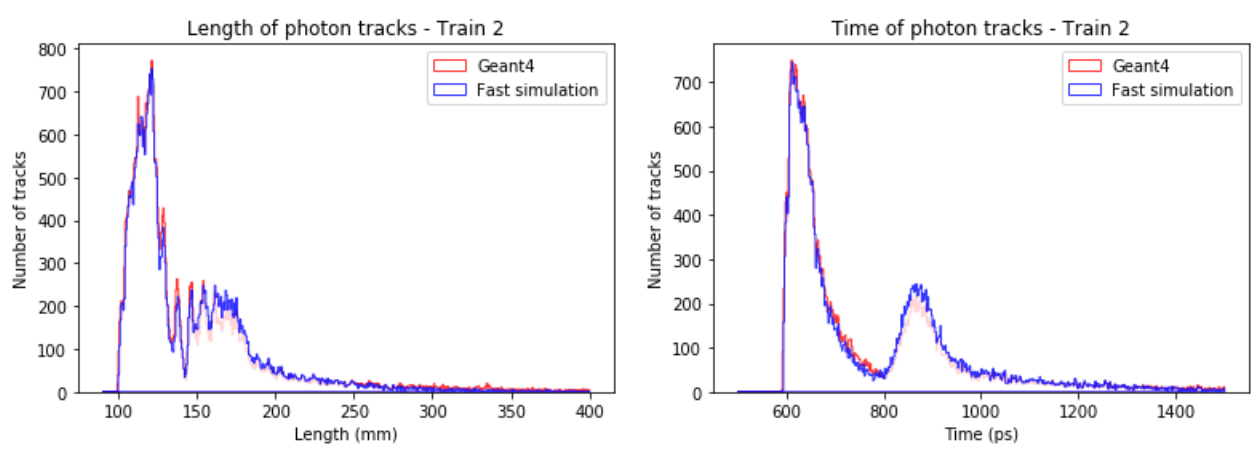

Figure 6. Histogram of photon track lengths and times - train 2.

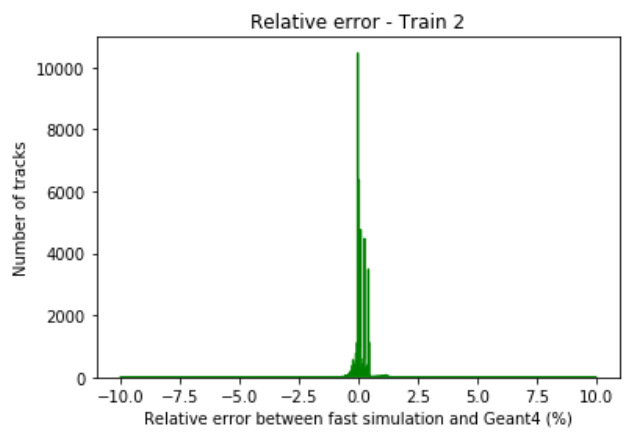

Figure 7. Relative error $\left(L-L_{\text {geant }}\right) / L_{\text {geant }}$ for photon tracks.

\subsection{Speed of the simulations}

By generating initially $3.3 \cdot 10^{6}$ photons using a computer with Intel Core i7-2600K 3,4 GHz, 32GB RAM, we get the following results for the duration of the simulations:

$$
\text { Geant4 simulation: } ~ 11.5 \text { minutes vs Fast simulation: } \sim 3 \text { seconds. }
$$

The fast simulation is then about 200 times faster.

\section{Application of the fast simulation: analysis of time resolution}

In the experiment, the proton trajectory and momentum is determined by the Silicon Tracker (SiT). This is a rectangular grid of size $17,6 \mathrm{~mm} \times 20 \mathrm{~mm}$, composed of pixels of size 50 $\mu \mathrm{m} \times 250 \mu \mathrm{m}$ [13]. The fast simulation allows us to generate a high number $N_{p}$ of proton trajectories, which emerge randomly from the SiT with precise coordinates. We consider protons entering into train 2 only. Each proton generates a number $N$ of photons, with $N$ following a Poisson distribution with the mean $\mu \approx 3300$ (given by eq.1). The result is shown in fig. 8 , for $N_{\mathrm{p}}=10^{5}$.

The fact that the minimal arrival time is the same for all bars is in agreement with the idea behind the geometrical shape of the ToF detector. The amplitude of the final signal (denoted as $A$ ) can be approximated by the size of the peak of the photon distribution. Due to the edge 


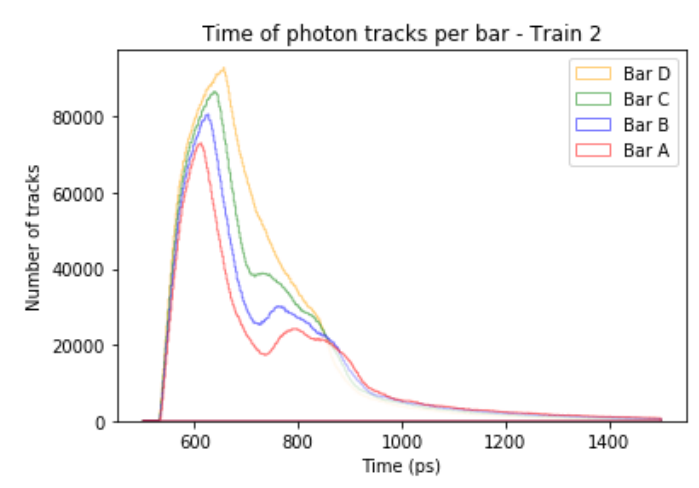

Figure 8. Time distributions for each bar - train 2.

effect, the amplitude of bar $\mathrm{D}$ is greater than the one of bar $\mathrm{A}: A_{\mathrm{A}}<A_{\mathrm{B}}<A_{\mathrm{C}}<A_{\mathrm{D}}$. From the amplitude, we can calculate the weight/time resolution $\sigma_{\mathrm{t}}$ of each bar, which is given by the approximative relation $\sigma_{\mathrm{t}} \propto 1 / \mathrm{A}$. We present in the following table the relative values of the amplitude and time resolution for each bar of train 2, the reference being bar A.

\begin{tabular}{|c|c|c|c|c|}
\hline Bar & A & B & C & D \\
\hline Amplitude $A$ & 1 & 1.11 & 1.19 & 1.27 \\
\hline Time resolution $\sigma_{\mathrm{t}}$ & 1 & 0.90 & 0.84 & 0.79 \\
\hline
\end{tabular}

Moreover, we represent in the following figure the time distributions in train 2, for different positions of proton trajectories (along $y$-coordinate, with a range of values $\pm 250 \mu \mathrm{m}$ ). We observe a local maximum peak around $650 \mathrm{ps}$ for each $y$-coordinate. The peak amplitudes increase when $y$ decreases; this is due again to the edge effect (proton trajectories with small $y$ are closer to the detector edge).

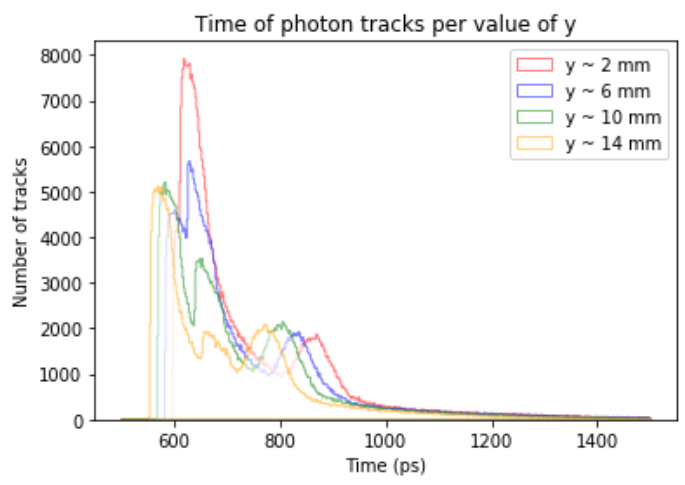

Figure 9. Time distributions for different $y$-coordinate of proton trajectories - train 2.

Finally, we can study the time resolutions of the different bars, the reference being the bar A of train 1. We illustrate it in fig.10, with the notations used in fig.2. We see that, independently of train, bar D has better time resolution than bar A (due to higher amplitude of the signal); for example, for train 4 , the resolution of bar $\mathrm{A}$ is $\sim 0.6$ while the resolution of bar $\mathrm{D}$ is $\sim 0.4$. Moreover, the train 3 has the best time resolution of the trains. 


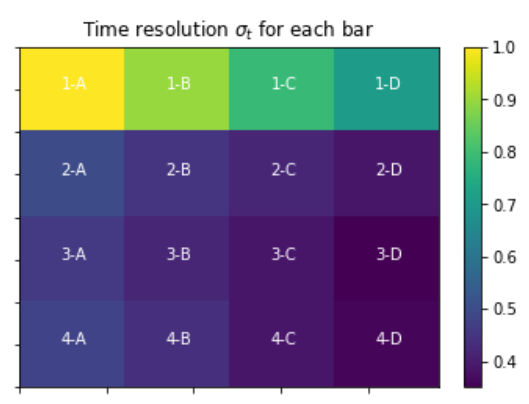

Figure 10. Relative time resolutions of the different bars of the ToF detector.

\section{Conclusion}

We presented the fast modeling of the AFP Time-of-Flight detector, that uses the geometrical method. The distributions of lengths obtained by the fast simulation and Geant 4 have the same shape, with relative difference $<2 \%$. An advantage of the fast simulation compared to Geant 4 is the speed-up of a simulation; it is about 200 times faster, and allows one to generate a higher number of proton trajectories, thus improving statistics. The code of the fast simulation, written in Python 3, is available at: https://github.com/olivierrousselle/ Fast-simulation-AFP. Users can choose the train, the number and positions of proton trajectories they want to model, and other parameters as the material of bars.

We suggest that such a fast simulation with Python and Numba could be used to model other types of ToF detectors; it seems more convenient and faster than Geant4, provided that physical processes aren't too complex.

\section{Acknowledgment}

Tomas Sykora gratefully acknowledges the support through LM2015056 and LTT17018 of MEYS.

\section{Bibliography}

[1] S.Agostinelli et al., Geant4-a simulation toolkit, Nuclear Instruments and Methods in Physics Research Section A, 506 (2015)

[2] ATLAS Collaboration, ATLAS Forward Proton - Technical Design Report, CERN, ATLAS-TDR-024, 009 (2015)

[3] L. Nozka, Design of Cherenkov bars for the optical part of the time-of-flight detector in Geant4, Optical Express, 22 (2014)

[4] M. Dyndal, T. Schoeffel and T. Sykora, Fast Cherenkov model of optical photons generation and transportation, ATL-SOFT-PUB-001 (2017)

[5] A. Marowka, Python accelerators for high-performance computing, The Journal of Supercomputing, 74 (2018)

[6] S. Kwan Lam , A. Pitrou and S. Seibert, Numba: a LLVM-based Python JIT compiler, Proceedings of the Second Workshop on the LLVM Compiler Infrastructure in HPC, 7 (2015)

[7] L. Nozka et al., Construction of the optical part of a time-of-flight detector prototype for the AFP detector, Optical Express, 24 (2016) 
[8] K. Jirakova, Fast simulation of Cherenkov photons transmission through the optical part of the AFP detector, bachelor thesis Palacky University Olomouc (2017)

[9] T. Komarek, Simulation of optical part of high-energy particle time-of-flight detector and comparison with data, master thesis Palacky University Olomouc (2016)

[10] https://www.heraeus.com/media/media/hca/doc_hca/products_and_solutions_8/optics/ Data_and_Properties_Optics_fused_silica_EN.pdf

[11] M. Albrow et al., Quartz Cherenkov counters for fast timing: QUARTIC, JINST 7 P10027 (2012)

[12] O. Rousselle, Fast simulation of the ATLAS Forward Proton detector, CERN Summer student report (2019)

[13] P. Erland, ATLAS Forward Proton Detectors, Status and Plans, Proceedings VERTEX2018 (2018) 